\title{
DNA transformation-the law of the tetrad
}

\section{Opinion}

One of the most basic interpretations of the Law of the Tetrad is that all outward communication as information is received inwardly (DNA is at the center of a cell), and modifies the organization of the internal system (etheric body), altering its vibratory frequency and outer appearance (material structure). DNA is composed of a liquid crystalline substance that's both etheric and material in nature, and acts as a medium for the transfer and decoding of information between the etheric holographic fields of astral light, and the material body. Because DNA is primarily composed of etheric substance (liquid light), it's modulated through an exchange of information that works intuitively on the etheric body, which is what's used to re-inform the cellular structure and specialization process of the material-molecular body. The exchange of information occurs within the inner core of all things as a form of receiver that picks up on waves being transmitted through the ether, which modifies the "informational structure" of the core. Just as the Pineal gland acts as the receiver and decoder of information from higher realms, the DNA of the body acts as a receiver and decoder for complementary information that instantaneously changes the state of every cell (energetic entanglement) through the integration and assimilation, altering the whole body based on the energetic state of the etheric body as the subtle electromagnetic field of information. The subtle realm of both the material body and the entire material realm, exist and function in a unified and holistic manner, as if they're "one organism", so all of what we call "communication" doesn't take place in "space and time" ( $4^{\text {th }}$ dimension $)$, but comes as an instantaneous shift that happens everywhere in the body at once. Vibratory information as waves are reflected over the surface of the mind-field (bio-field) that surrounds, envelopes, and permeates the physical body (electromagnetic field) and is absorbed (inducted and sorted) and received internally by the DNA-Pineal, and serves to reprogram the etheric hologram as the internal reality that arises naturally in the imagination. Through the corresponding operations of both the Pineal and DNA, the "mind and body", and the entire outer reality of which they exist as a part, are always in-sync as "one organism".

The phantom (Holy Ghost) is a holographic pre-image of biostructures that accompanies all biological forms and exists as a whole registry of wave-copies and dynamic matrixes of information as a convoluted continuum used for organizing, constructing, regenerating, and animating them in an automated fashion. This holographic preimage as the informational-fields used to structure and regenerate the body is known in Spiritual Sciences as the etheric body, which is the twin or double of the material body as a hologram or 3-D, spatial blueprint of it. This etheric hologram is composed of astral light as essence or biophotons that both receive and transmit information carried on waves through the astral plane of the Earth as morphogenetic fields of the instinctual plane of the collective unconscious. The basic Law of the Tetrad, is when two fields (Monads) vibrating at the same frequency merge into and blend with each other (resonant induction), forming an interference pattern (Dyad), that acts to cancel out some attributes, while matching and amplifying other ones, modifying the overall vibration into a new one as their unique combination (triad) that forms coherence as a new pattern or offspring that re-informs and alters the etheric body, which modifies the cellular regeneration of
Volume 7 Issue 3 - 2018

\author{
Linda Gadbois \\ Spiritual Scientist, USA
}

Correspondence: Linda Gadbois, Spiritual Scientist, Colorado Springs, CO 80915, USA, Tel 3037269344;

Email Dr.lindagadbois@outlook.com

Received: January 18, 2018 | Published: May 10, 2018

the physical body (Tetrad). The Law of Tetrad represents when nonlocal information enters into the local apparatus through resonance, and reformulates it into a coherent pattern as an internal image (sign) that's "reflected" outward as a projection forming the physical equivalent as its expression. The information is "recorded" (like a mirror image) on the physical DNA used to organize electromagnetic fields as organizational structures of proteins into the pattern or configuration of the frequency, which forms the etheric body as a holographic template for organizing the molecular structure of the organism. The etheric hologram as a biofield acts as both a transmitter and receiver of waves of information that are of the same form or frequency. The incorporation of diverse information of a similar nature that can be synthesized into the cellular programming evolves it to new structures, forms, and ways of being. It forms a greater and more diverse range of self-expression.

DNA is a biocomputer as a quantum hologram that's a convoluted liquid crystalline structure that's an intricate dynamic matrix of information that's always morphing based on how it's being programmed with new integrated information. It's always receiving information from other morphic fields around it, interpreting that information through a process of adapting it to its existing structure, changing its meaning, imprinting and recording the modified version of the information etherically, altering its structure (vibration) accordingly, while simultaneously radiating, emitting, and transmitting it. Vibration as a field of information produces an electromagnetic field as a holographic form that takes information as thoughts and words, and turns it into a 3-D form comprised of subtle energy (invisible) that becomes the invisible force as a field that organizes and holds matter together in a biological living system.

Scientists have now discovered that what they originally thought was "junk DNA" ( $98 \%$ of it), is actually more of a memory bank that operates much like the human mind does, and is always exchanging energy as information with everything else, and works off the same rules as grammar and language does. Its encoded information is linguistic in nature, and contains dual wave-forms as both optical and acoustic. It works through speech as words and sentences that form images in the imagination as the meaning of the words. Words as a sound vibration form into patterns of light as the image inherent in the word, or that the word represents in terms of what it means. As we speak us simultaneously form and internal reality of what we're talking about. Words form images, and sentences form scenarios or a chainof-association as whole realities. A reality produces an experience of that reality as its reason and meaning. The meaning things have for us produce the stories we tell ourselves about things as a way of making 
sense of them. Our stories as an internal narrative exists as a form of "theme" produced by our mental paradigm (vibratory structure of our mind as a dynamic matrix of associated ideas) give us a consistent and cohesive experience of reality. Everything that exists happens for a reason because all things are called forth into reality through thoughts (internal speech), which are the cause of all forms.

Our DNA, like our mind and body (subconscious), is programmed linguistically and through visual imagery. One of the fundamental laws is the Law of Cause and Effect, in which every effect as a material form has a cause and a reason for being here. Nature operates out of the Law of Conservation, and never produces excess or waste of any kind. Everything exists for a reason and has a purpose. The phantom effect as vibration that produces an electromagnetic field as the holographic pattern inherent in the vibration, is based on information as both verbal and visual information which exists in its most primary state as thoughts. As we think and imagine, our thoughts as a virtual reality gives us an internal experience of them, where we are literally programming our DNA as our subconscious mind to produce the reality of those thoughts as an outward, corresponding material experience.

The ongoing programming of our genes doesn't change our physical qualities and characteristics, but rather works topologically, where it changes our inner constitution. As we develop by programming ourselves with our own thoughts and the thoughts of others (speaking), our character is developed without changing our body or general structure. We program our genes with all levels of our being through our thoughts, feelings, emotional states, and behavioral dynamics that all form different aspects of the same cohesive reality. As we change our inner being as our mental paradigm, the physical world itself doesn't change, but how it "appears to us" changes. Our perception of it can change quite drastically. The perspective we take and the perception we form from that perspective, create our outer reality as our experience of it based on resonance. Experiences that we form that are intense or have a strong emotional component make up what we can call our actual memory as long term memory that we continue to create out of forming all of our other life experiences to be of a similar nature, are also the memory that programs our genes and are passed down generation to generation as inherited tendencies. We also act to populate the astral field of morphic resonance with those memories, making them available to anyone that's of a similar nature to us, and who can therefore act as a receiver and processor for those memories as their "own thoughts".

Morphic resonance works on the same principles as quantum entanglement and quantum coherent system assembly, where once two fields and particles blend and form into a variant, both continue to operate as one, as a whole system of complementary opposites. What we connect with, feel a sense of chemistry with, or form a pronounced reaction to in any way, is what seemingly exists outside of ourselves as a complementary opposite to the same pattern as a behavioral dynamic. We can only "see" (perceive) in everything else what's of the same nature (vibratory pattern) as we are. All comprehension comes as a form of resonance, as the vibratory pattern that forms our mental filters that we look through that only allow us to see and respond to what's of the same pattern as opposite roles in a cohesive system. We only alter our own vibration as a mental state by incorporating variable information within the same frequency, re-informing our overall system with new variables. For this reason most evolution is a very gradual process that takes place over long periods of time, and by acquiring different bodies with different characteristics, in different conditions and circumstances, that allow us to develop in new ways. We can facilitate and greatly speed up our own evolution by undertaking a constant form of learning by continuously providing ourselves with new information that we absorb and integrate, producing different types of experiences that become the memory that upgrades our paradigm and changes our character, our basic demeanor and behaviors, and correspondingly our perception of the outer world. As we change internally through the integration of new information that produces new perceptual filters and experiences that become memory as our vibratory structure, our outer reality as our perception of it, changes accordingly.

All information as knowledge, has dual properties as both acoustic and optical that together form the "phantom effect" as an electromagnetic field that organizes light as essence into a form that serves as a blueprint, or 3-D spatial map for generation, cell differentiation, and spatially locating within the whole system as a growth and development process. Transmission of information as the teleportation of the hologram will produce the physical equivalent as an organizational process. It simultaneously attracts and organizes into a holistic system whatever is "like it" (same type of information), while repelling and not noticing what's not. As we think, both in words and pictures, we produce the electromagnetic field of those thoughts as a virtual reality that becomes the magnetic force for gathering, organizing, and producing its correspondence as an outer reality that gives us the experience of it. Our thoughts are the creative force of both ourselves and our perception of reality because the same vibration as a holographic pattern is producing both. We are always the ones creating and evolving ourselves through our thoughts which form our entire reality. The same vibration as a pattern structures the internal and the external to be of the same nature and convey the same meaning.

\section{Acknowledgments}

None

\section{Conflict of interest}

Authors declare there is no conflict of interest. 